Check for updates

Cite this: RSC Adv., 2019, 9, 18285

\title{
Using magnetic levitation for density-based detection of cooking oils $\uparrow$
}

\author{
Chengxian Zhu, ${ }^{a}$ Lei Gao, ${ }^{a}$ Yaojie Han, ${ }^{\text {a }}$ QiRan OuYang, ${ }^{a}$ Lijun Zong, ${ }^{a}$ Lin Li, (D) a \\ Chengwu Zhang, ${ }^{a}$ Jinhua Liu, ${ }^{a}$ Hai-Dong Yu (DD *ab and Wei Huang ${ }^{a b}$
}

Adulterated cooking oils and the repeated use of frying cooking oils are harmful to human health. Current techniques for the quality control of cooking oils such as gas chromatography and high performance liquid chromatography usually require expensive facilities, and they are complicated to operate. This paper describes a simple technique that uses magnetic levitation (MagLev) to analyse cooking oils based on density. We have demonstrated the application of MagLev to detect the quality of cooking oil by simulating adulterated sesame oil using peanut oil and frying soybean oil for different times. We have also demonstrated the use of MagLev to differentiate secondhand cooking oil from certified cooking oil. MagLev provides a portable and inexpensive method for the on-site inspection of cooking oils, and it may be extended to many other applications in food safety, environmental monitoring, medical diagnosis, and so on. The advantages of high sensitivity, low cost, and convenience of operation make MagLev especially useful for in situ applications in resource-limited settings.

Received 3rd April 2019

Accepted 4th June 2019

DOI: $10.1039 / c 9 r a 02516 b$

rsc.li/rsc-advances dealers sell inferior cooking oils, cooking oils blended with other cheap oils (such as peanut oil, whose price is half that of sesame oil), or use frying cooking oils repeatedly to maximize profits. Adulterated cooking oils and the repeated use of frying cooking oils not only compromise the quality of the food but also pose a potential hazard to human health. ${ }^{8}$

Current techniques for the quality control of cooking oils include gas chromatography, ${ }^{9-11}$ gas chromatography-mass spectrometry, ${ }^{\mathbf{1 2}, 13}$ high performance liquid chromatography (HPLC), ${ }^{\mathbf{1 4}}$ and nuclear magnetic resonance spectroscopy (NMRS). ${ }^{15-17}$ However, these techniques are complicated and time-consuming, and they require expensive facilities and trained personnel. Therefore, there is an urgent need to develop a simple, inexpensive, portable, and rapid method for the onsite detection of various cooking oils.

Magnetic levitation (MagLev), an emerging analytical technique that maps the density of a diamagnetic object by its position levitated in a paramagnetic solution, ${ }^{\mathbf{1 8}}$ may answer to this demand. The technique is compatible with most types of diamagnetic objects with densities of $0.8-3 \mathrm{~g} \mathrm{~cm}^{-3}$ with a high accuracy ranging from $\pm 0.0002 \mathrm{~g} \mathrm{~cm}^{-3}$ to $\pm 0.02 \mathrm{~g} \mathrm{~cm}^{-3} \cdot{ }^{19}$ Due to its portability, low cost, simplicity to operate, and precise measurement through visual readout, this density-based technique has found wide applications in separation, ${ }^{\mathbf{2 0}}$ selfassembly, ${ }^{21,22}$ forensics, ${ }^{23}$ quality control, ${ }^{24}$ food safety, ${ }^{25}$ and bioanalysis. ${ }^{26-29}$ For example, MagLev has been used to perform nondestructive quality control of plastic components ${ }^{24}$ and determine nutritionally relevant properties of food and salinity of water. ${ }^{25}$ When considerations of cost, simplicity, portability, and requirement for low sample volume or irregular sample anstitute of Advanced Materials (IAM), Key Laboratory of Flexible Electronics (KLOFE), Jiangsu National Synergetic Innovation Center for Advanced Materials (SICAM), Nanjing Tech University (NanjingTech), 30 South Puzhu Road, Nanjing 211816, Jiangsu, PR China. E-mail: iamhdyu@njtech.edu.cn

${ }^{b} X i$ 'an Institute of Flexible Electronics, Northwestern Polytechnical University, 127 West Youyi Road, Xi'an 710072, Shaanxi, PR China

$\dagger$ Electronic supplementary information (ESI) available. See DOI: 10.1039/c9ra02516b 
shape outweigh the need for analysing precise chemical composition of samples, MagLev is particularly useful. ${ }^{25}$ With its simple setup and operation, MagLev is especially suitable for in situ or point-of-care applications ${ }^{\mathbf{3 0 , 3 1}}$ in resource-constrained settings (e.g., supermarkets, restaurants, mines, archaeological sites, field operations, and home healthcare).

In this study, we demonstrated the use of MagLev for density-based detection of cooking oils. We mixed sesame oil with peanut oil and determined the content of sesame oil by the levitation height of the oil droplets in MagLev, showing the potential of MagLev for the quality inspection of adulterated cooking oils. We heated soybean oil for up to seven hours and determined the heating time by the levitation height of the oil droplets in MagLev, showing the potential of MagLev for the quality inspection of frying cooking oils with repeated use. We also demonstrated the use of MagLev to distinguish secondhand cooking oil from certified cooking oils by the levitation height of the oil droplets. Compared with traditional methods, MagLev provides an easy approach with low cost and portability for the rapid detection of cooking oils. This approach may find wide applications in quality control, food safety, and so on.

\section{Experimental}

\section{Materials and instruments}

NdFeB magnets $(\sim 0.3 \mathrm{~T}, 50 \mathrm{~mm} \times 50 \mathrm{~mm} \times 25 \mathrm{~mm})$ were purchased from the Genchang magnet material Co., Ltd, China. Manganese chloride $\left(\mathrm{MnCl}_{2} \cdot 4 \mathrm{H}_{2} \mathrm{O}, \mathrm{AR}\right)$ was purchased from the Adamas-Beta Co., China. Methanol (AR) was purchased from the Shanghai Lingfeng chemical reagent Co., Ltd, China. Tween 20 (AR) was purchased from the Shanghai Titan chemical Co., Ltd, China. Sesame oil, soybean oil, and peanut oil were purchased from the local supermarket. Pure soybean oil without any additives was obtained by squeezing soybeans in the local mill of cooking oils. Secondhand cooking oil (soybean oil) was collected from a fried food restaurant and filtered in the laboratory. A magnetic stirrer (C-MAG HS7, IKA, Germany) was used to heat the cooking oils. The deionized water $(18.4 \mathrm{M} \Omega \mathrm{cm})$ used for all the experiments was obtained from a Milli-Q system (Millipore, Bedford, USA). All photographs were taken with a digital camera (Canon 700D, Japan).

\section{Theory of MagLev}

As shown in Fig. 1, the MagLev device consisted of two identical NdFeB magnets, with like poles facing each other, and a transparent container filled with paramagnetic solution (e.g., $\mathrm{MnCl}_{2}$ solution). The magnetic force, $\vec{F}_{\text {mag }}$, caused by the magnetic field and the gravitational force (corrected for the effect of buoyancy), $\vec{F}_{\text {g }}$, acting on the diamagnetic sample (e.g., the oil droplet) are given as:

$$
\begin{gathered}
\vec{F}_{\mathrm{mag}}=\frac{\left(\chi_{\mathrm{s}}-\chi_{\mathrm{m}}\right)}{\mu_{0}} V(\vec{B} \cdot \vec{\nabla}) \vec{B} \\
\vec{F}_{\mathrm{g}}=\left(\rho_{\mathrm{s}}-\rho_{\mathrm{m}}\right) V \vec{g}
\end{gathered}
$$
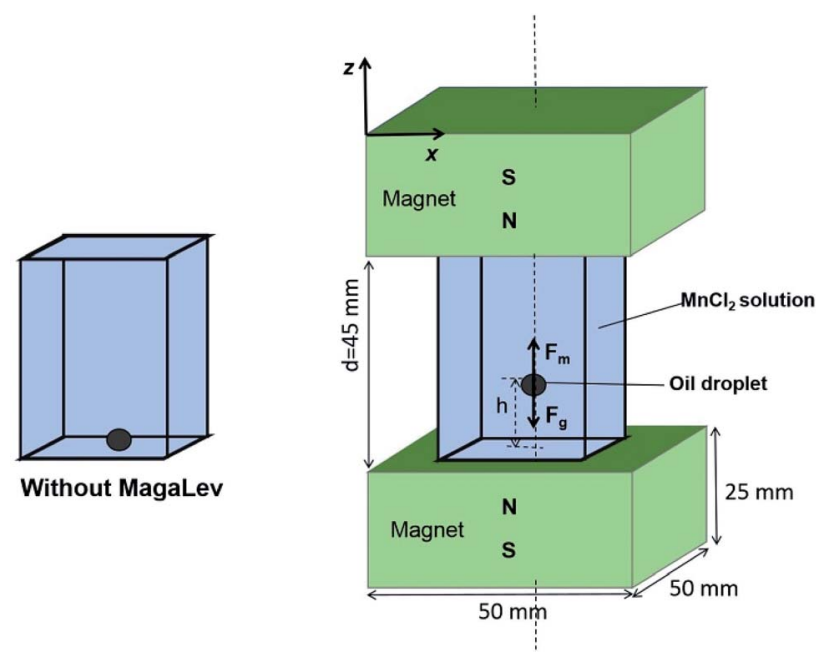

Fig. 1 Schematic representation of the experimental setup of magnetic levitation (MagLev). A container filled with $\mathrm{MnCl}_{2}$ solution was placed between two indistinguishable NdFeB magnets $(50 \mathrm{~mm} \times$ $50 \mathrm{~mm} \times 25 \mathrm{~mm}$ ), aligned vertically, $45 \mathrm{~mm}$ apart, with like poles facing each other. The droplet of cooking oil was levitated in the MagLev device due to the balance of the gravitational force, $\vec{F}_{\mathrm{g}}$, and the magnetic force, $\vec{F}_{\text {mag }}$.

where $\chi_{\mathrm{s}}$ and $\chi_{\mathrm{m}}$ are the magnetic susceptibilities of the diamagnetic sample and the paramagnetic solution, respectively, $\mu_{0}$ is the magnetic permeability of free space, $V$ is the volume of the sample, $\vec{B}$ is the magnetic flux density, $\rho_{\mathrm{s}}$ and $\rho_{\mathrm{m}}$ are the densities of the diamagnetic sample and paramagnetic solution, respectively, and $\vec{g}$ is the vector of gravity. ${ }^{19}$ The sample reaches its equilibrium position when the net force acting on the sample is zero, i.e.:

$$
\vec{F}_{\mathrm{g}}+\vec{F}_{\mathrm{mag}}=\left(\rho_{\mathrm{s}}-\rho_{\mathrm{m}}\right) V \vec{g}+\frac{\left(\chi_{\mathrm{s}}-\chi_{\mathrm{m}}\right)}{\mu_{0}} V(\vec{B} \cdot \vec{\nabla}) \vec{B}=0
$$

These two forces balance each other only along the centerline. From eqn (3), it can be determined that the levitation height of the sample is correlated linearly with its density:

$$
h=\frac{\left(\rho_{\mathrm{s}}-\rho_{\mathrm{m}}\right) \vec{g} \mu_{0} d}{\left(\chi_{\mathrm{s}}-\chi_{\mathrm{m}}\right) 4 B_{0}{ }^{2}}+\frac{d}{2}
$$

where $B_{0}$ is the maximum magnetic flux density along the centerline of the device, and $d$ is the distance between the two magnets. ${ }^{19}$

\section{Procedure of detection}

Because the sample in this experiment was an oil droplet, the paramagnetic solution had to be inert to oil, and it could not dissolve or swell from the oil droplet. ${ }^{25}$ Additionally, the density of the paramagnetic solution had to be close to the density of the oil droplet. For these purposes, the paramagnetic solution was prepared by dissolving $\mathrm{MnCl}_{2} \cdot 4 \mathrm{H}_{2} \mathrm{O}$ in a mixture of water and methanol, with the addition of Tween 20 to remove air bubbles. Typical solubilities of the major components of vegetable oils in methanol were $0.5-5 \%(v / v) .{ }^{32,33}$ The miscibility of 
oils with methanol/water mixtures used in our experiments was below $0.5 \%(\mathrm{v} / \mathrm{v})$. Also, the amount of Tween 20 added in the paramagnetic solution was very small (1 droplet in $100 \mathrm{~mL}$ solution). Thus, we expected that they did not interfere much with analysis on the time scale of the experiment (within 15 seconds). The methanol/water mixture ratio was optimised to match the density of the paramagnetic solution with that of the oil to be detected (the density of the paramagnetic solution decreased with the larger methanol/water mixture ratio). The concentration of the paramagnetic solution was optimised to allow the levitation of all the oil droplets (a higher concentration enabled a wider range of density measurement). The lowest concentration that allowed the levitation of all the oil droplets was selected for high-sensitivity detection. ${ }^{19}$

For the detection of cooking oils, an oil droplet was added into the container filled with $\mathrm{MnCl}_{2}$ solution, which was sealed with parafilm, and the container was placed in the MagLev device, with a ruler affixed to the device. The size of the oil droplets was $\sim 2 \mathrm{~mm}$ in diameter. After the oil droplet was stably levitated, a photograph of the MagLev device was taken with a digital camera and the levitation height of the oil droplet was read by the ruler. For practical applications, while the density of each kind of cooking oil is roughly within a small range, the solution containing the appropriate amount of $\mathrm{MnCl}_{2}$ can be prepared in advance according to the cooking oil to be detected. In this work, sesame oil, peanut oil and soybean oil have been selected as examples.

\section{Detection of mixed cooking oils}

For the detection of adulterated oil, sesame oil and peanut oil were mixed with the proportions of sesame oil in the mixed oil of $0 \%, 10 \%, 30 \%, 50 \%, 70 \%, 90 \%$, and $100 \%$. The individual droplets of the mixed oil with different proportions of sesame oil were levitated in $0.1 \mathrm{M}, 0.2 \mathrm{M}$ and $0.4 \mathrm{M} \mathrm{MnCl}_{2}$ solution in $63 \%$ methanol and $37 \%$ water (v/v) in the MagLev device. Each measurement was repeated five times. The photographs of the MagLev device were taken, and a calibration curve between the proportion of the sesame oil in the mixed oil and the levitation height of the droplets of the mixed oil was plotted.

\section{Detection of heated soybean oil}

For the detection of the repeated use of frying cooking oil, two types of soybean oil were purchased from local supermarkets (purchased soybean oil) and a cooking oil mill (pure soybean oil without any additives). A magnetic stirrer was used to heat the two types of soybean oil at $200{ }^{\circ} \mathrm{C}$ for $7 \mathrm{~h}, 2 \mathrm{~mL}$ of which was taken out every hour. Then seven droplets of purchased soybean oil heated for $1 \mathrm{~h}, 2 \mathrm{~h}, 3 \mathrm{~h}, 4 \mathrm{~h}, 5 \mathrm{~h}, 6 \mathrm{~h}$, and $7 \mathrm{~h}$ were levitated in $0.6 \mathrm{M} \mathrm{MnCl}_{2}$ solution in $80 \%$ methanol and $20 \%$ water (v/v), and seven droplets of pure soybean oil with different heating time were levitated in $0.2 \mathrm{M} \mathrm{MnCl}_{2}$ solution in $67 \%$ methanol and $33 \%$ water $(\mathrm{v} / \mathrm{v})$ in the MagLev device. Each measurement was repeated five times. The photographs of the MagLev device were taken and a calibration curve between the heating time and the levitation height of oil droplets was plotted.

\section{Detection of secondhand cooking oil}

The secondhand cooking oil was collected from a fried food restaurant and filtered simply in the laboratory. The raw material of this oil was soybean oil. Individual droplets of certified soybean oil and secondhand cooking oil were levitated in $0.5 \mathrm{M} \mathrm{MnCl}_{2}$ solution in $80 \%$ methanol and $20 \%$ water $(\mathrm{v} / \mathrm{v})$ in the MagLev device. Each measurement was repeated five times. The photographs of the MagLev device were taken and the levitation heights of secondhand cooking oil and certified soybean oil were compared.

\section{Results and discussion}

\section{MagLev-based detection of adulterated cooking oil}

For the detection of adulterated oil, we used a demonstration of with MagLev to determine the content of sesame oil in a mixed oil of sesame oil and peanut oil (because the price of peanut oil is half of that of sesame oil, peanut oil is often used in adulterated sesame oil). Individual droplets of the mixed oil with $0 \%, 10 \%, 30 \%, 50 \%, 70 \%, 90 \%$, and $100 \%$ of sesame oil were levitated in the MagLev device, and their levitation heights were compared. The oil droplets were stably levitated in the solution within $15 \mathrm{~s}$ after the container was placed in the MagLev device. Because the container was sealed, the effect of the evaporation of the solvent on the levitation height of the oil droplets could be ignored in $15 \mathrm{~s}$. It could be observed that the levitation height of the oil droplets decreased with increasing proportions of the sesame oil in the mixed oil of sesame oil and peanut oil (Fig. 2a). When the proportion of sesame oil was $0 \%$, the oil droplet was peanut oil, which was levitated in the upper part of the container, indicating that the density of the peanut oil was less than that of the paramagnetic solution. ${ }^{18}$ When the proportion of sesame oil was $100 \%$, the oil droplet was sesame oil, which was levitated in the lower part of the container, indicating that the density of sesame oil was greater than that of the paramagnetic solution. ${ }^{19}$ Because the density of sesame oil was greater than the density of peanut oil, the density of the mixed oil increased with the increasing proportion of sesame oil in the mixed oil, resulting in the decreased levitation height of the oil droplets.

As shown in Fig. 2b, the levitation height of the oil droplets decreased linearly with the increasing proportions of sesame oil in the mixed oil of sesame oil and peanut oil, which was consistent with eqn (4). ${ }^{19}$ The levitation of the oil droplets was greatly affected by the concentration of the $\mathrm{MnCl}_{2}$ solution. When the concentration of $\mathrm{MnCl}_{2}$ solution was $0.1 \mathrm{M}$, all the droplets of mixed oil sank to the bottom of container and could not levitate in the solution. When the concentration of $\mathrm{MnCl}_{2}$ solution was $0.4 \mathrm{M}$, all the oil droplets were levitated. However, the difference between the levitation heights of the oil droplets in $0.4 \mathrm{M} \mathrm{MnCl}_{2}$ solution was smaller than that in $0.2 \mathrm{M} \mathrm{MnCl}_{2}$ solution. When the solution allowed levitation of all the oil droplets, the correlation coefficients increased with larger concentration of $\mathrm{MnCl}_{2}$ solution, and the change in levitation height of the oil droplets decreased with larger concentration of $\mathrm{MnCl}_{2}$ solution (Fig. 2b). Thus, 0.2 $\mathrm{M}$ was selected here as the 

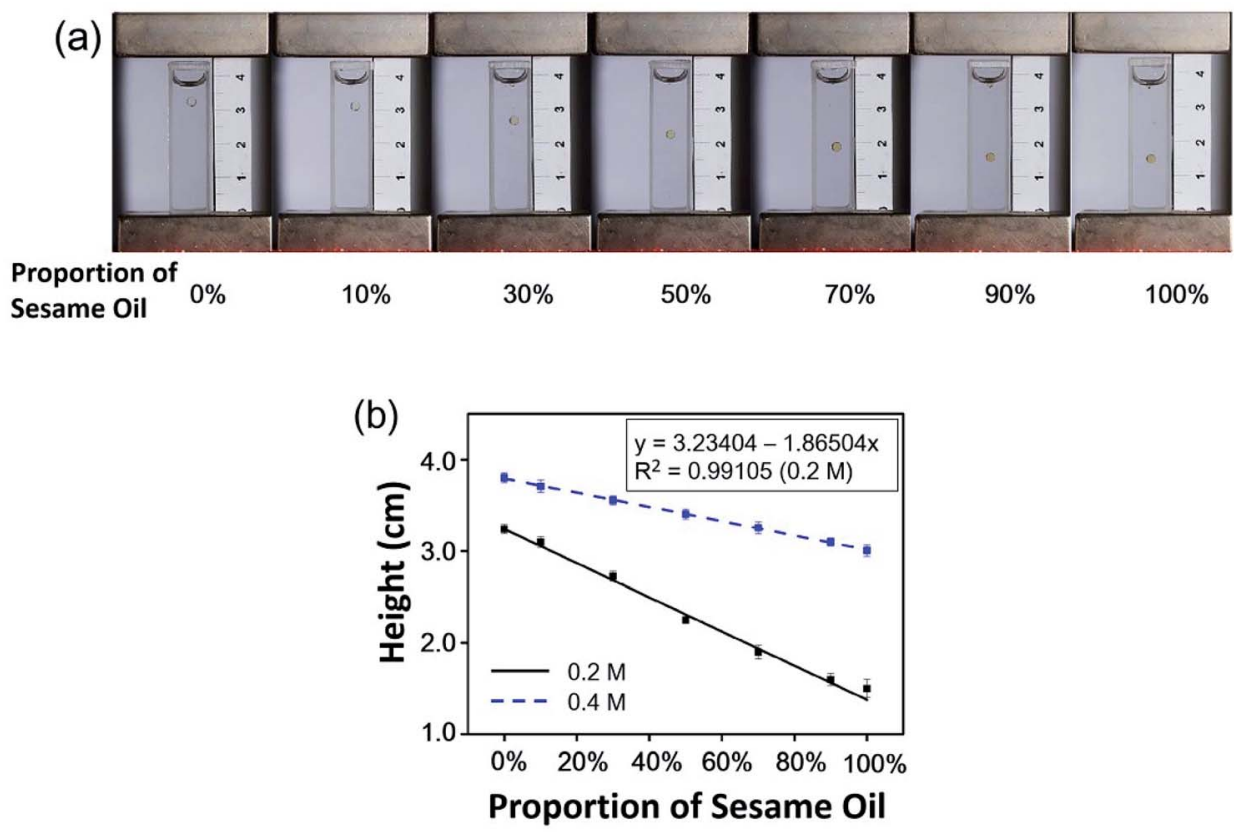

Fig. 2 Detection of mixed cooking oil using MagLev. (a) Photographs of the droplets of mixed oil of sesame oil and peanut oil with different proportions of sesame oil of $0 \%, 10 \%, 30 \%, 50 \%, 70 \%, 90 \%$, and $100 \%$ levitated in $0.2 \mathrm{M} \mathrm{MnCl}_{2}$ solution in the MagLev device. (b) Plots correlating the levitation height of the droplets of the mixed oil with the proportion of sesame oil in $0.2 \mathrm{M}$ and $0.4 \mathrm{M} \mathrm{MnCl}_{2}$ solution. Each data point is the mean of five analyses.

concentration of $\mathrm{MnCl}_{2}$ solution for high-sensitivity detection of adulterated oils. Therefore, we could derive the proportion of sesame oil in the mixed oil based on the levitation heights of the oil droplets. In this example, we demonstrated that MagLev could be used to determine the content of sesame oil in the mixed oil when the sesame oil was adulterated with the cheaper peanut oil. Similarly, we could adjust the paramagnetic solution and use MagLev to detect various types of adulterated cooking oils.

\section{MagLev-based detection of the repeated use of frying cooking} oil

For the detection of the repeated use of frying cooking oil, we used MagLev to determine the heating time of purchased soybean oil. The purchased soybean oils were heated for $1 \mathrm{~h}$, $2 \mathrm{~h}, 3 \mathrm{~h}, 4 \mathrm{~h}, 5 \mathrm{~h}, 6 \mathrm{~h}$, and $7 \mathrm{~h}$. Individual oil droplets with different heating time were levitated in the MagLev device. It can be observed that the levitation heights of the droplets of the purchased soybean oil decreased with longer heating time (Fig. 3a). For example, the droplet of purchased soybean oil heated for $7 \mathrm{~h}$ was levitated closer to the bottom magnet than the droplet of purchased soybean oil heated for $1 \mathrm{~h}$, indicating that the density of purchased soybean oil heated for $7 \mathrm{~h}$ was greater than that of purchased soybean oil heated for $1 \mathrm{~h}$. The droplets of purchased soybean oil with longer heating time were levitated closer to the bottom magnet, which means that the densities of the purchased soybean oil increased with longer heating time. As shown in Fig. 3b, the levitation height of the droplets of purchased soybean oil decreased linearly with the heating time, suggesting that the heating time of the purchased soybean oil could be derived from the levitation heights of oil droplets.

To avoid the effect of any additives in the purchased soybean oil, we also measured the levitation heights of the droplets of pure soybean oil (without any additives) heated for $1 \mathrm{~h}, 2 \mathrm{~h}, 3 \mathrm{~h}$, $4 \mathrm{~h}, 5 \mathrm{~h}, 6 \mathrm{~h}$, and $7 \mathrm{~h}$. It can be observed that the pure soybean oil showed a trend similar to the purchased soybean oil and that the levitation heights of the oil droplets decreased with longer heating time (Fig. 4a). When the pure soybean oil was heated for $1 \mathrm{~h}$, the oil droplet was levitated in the upper part of the container, indicating that the density of pure soybean oil heated for $1 \mathrm{~h}$ was less than that of the paramagnetic solution. When the pure soybean oil was heated for $7 \mathrm{~h}$, the oil droplet was levitated in the lower part of the container, indicating that the density of pure soybean oil heated for $7 \mathrm{~h}$ was greater than that of the paramagnetic solution. The droplets of the pure soybean oil were levitated closer to the bottom magnet with longer heating time, indicating that the density of the pure soybean oil increased with longer heating time. As shown in Fig. $4 \mathrm{~b}$, the levitation height of the droplets of pure soybean oil decreased linearly with the heating time, which suggested that the heating time of the purchased soybean oil could be derived from the levitation heights of the oil droplets.

It has been reported that cooking oil that has been overheated (i.e., heated for a long time) and the repeated use of frying cooking oil are harmful to human health and that the density of cooking oil increases with increasing heating time. ${ }^{8}$ The reason for this may be that the grease in the cooking oil under a high temperature with a long heating time will conduct thermal polymerization and thermal oxidation polymerization, 

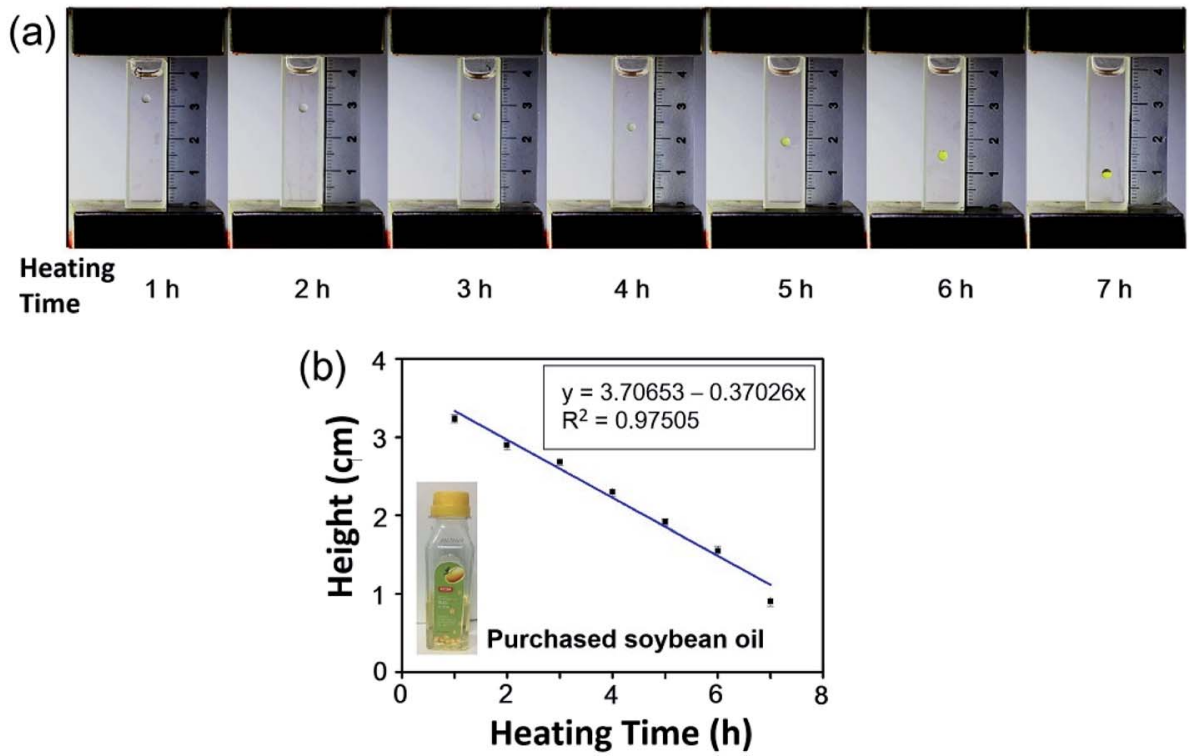

Fig. 3 Detection of heated purchased soybean oil using MagLev. (a) Photographs of the droplets of purchased soybean oil that was heated for $1 \mathrm{~h}, 2 \mathrm{~h}, 3 \mathrm{~h}, 4 \mathrm{~h}, 5 \mathrm{~h}, 6 \mathrm{~h}$, and $7 \mathrm{~h}$ levitated in the MagLev device. (b) Plot correlating the levitation height of the droplets of purchased soybean oil with the heating time.

producing cyclic polymer and glyceride dimer and other toxic components. Grease can also undergo hydrolysis and a condensation reaction to produce compounds with larger molecular weights such as ether compounds, resulting in the increase of the viscosity and density of the oil, which not only reduces the nutritional value, but also seriously harms the health of consumers. ${ }^{8}$
We also measured the initial densities of different cooking oils, such as sesame oil, peanut oil, purchased soybean oil, and pure soybean oil, and measured their densities after being heated at $200{ }^{\circ} \mathrm{C}$ for $3 \mathrm{~h}$ both by MagLev and the traditional method (mass divided by volume), as shown in Table 1. It was found that the results of the two methods were close to each other. As shown in Fig. 5, the bias between the

(a)

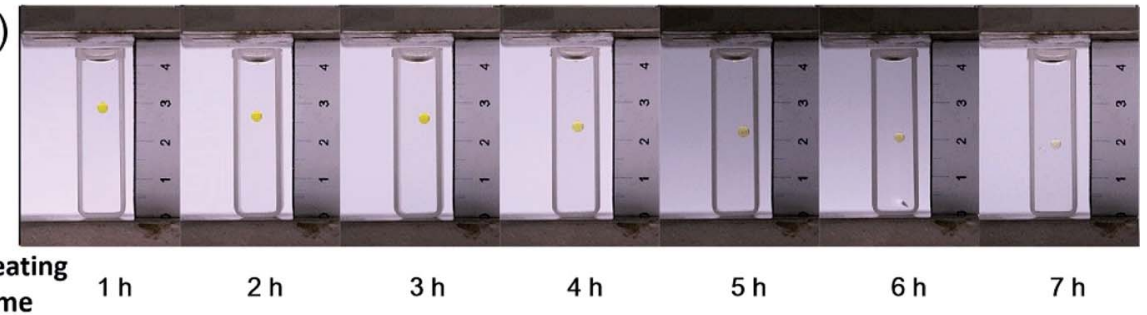

Heating

$1 \mathrm{~h}$

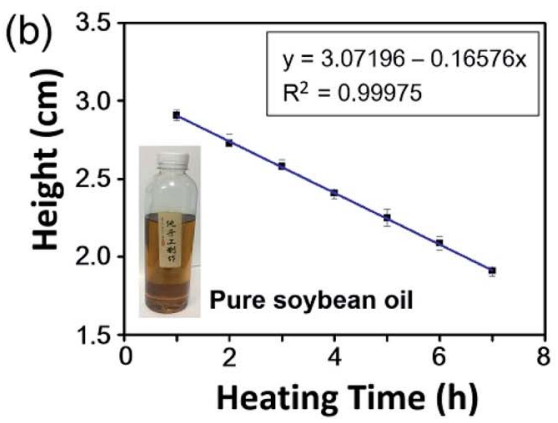

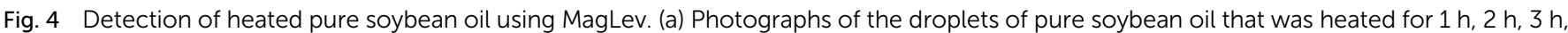

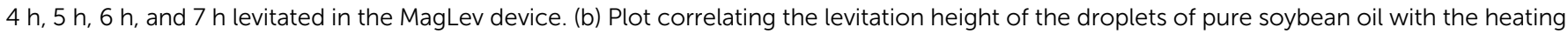
time. Each data point is the mean of five analyses. 
Table 1 Comparison of the initial densities of different cooking oils and the densities of these oils after being heated at $200{ }^{\circ} \mathrm{C}$ for $3 \mathrm{~h}$ measured by MagLev and the traditional method (mass divided by volume). Each data point is the mean of five analyses

\begin{tabular}{|c|c|c|c|c|}
\hline Methods & MagLev $\left(\mathrm{g} \mathrm{cm}^{-3}\right)$ & $\begin{array}{l}\text { Traditional method } \\
\left(\mathrm{g} \mathrm{cm}^{-3}\right)\end{array}$ & MagLev $\left(\mathrm{g} \mathrm{cm}^{-3}\right)$ & $\begin{array}{l}\text { Traditional method } \\
\left(\mathrm{g} \mathrm{cm}^{-3}\right)\end{array}$ \\
\hline Heating time (h) & 0 & 0 & 3 & 3 \\
\hline Sesame oil & $0.934 \pm 0.001$ & $0.933 \pm 0.001$ & $0.935 \pm 0.001$ & $0.934 \pm 0.002$ \\
\hline Peanut oil & $0.929 \pm 0.001$ & $0.927 \pm 0.002$ & $0.930 \pm 0.001$ & $0.929 \pm 0.001$ \\
\hline Soybean oil & $0.927 \pm 0.001$ & $0.925 \pm 0.002$ & $0.929 \pm 0.001$ & $0.927 \pm 0.002$ \\
\hline Pure soybean oil & $0.929 \pm 0.001$ & $0.928 \pm 0.001$ & $0.931 \pm 0.001$ & $0.930 \pm 0.001$ \\
\hline
\end{tabular}

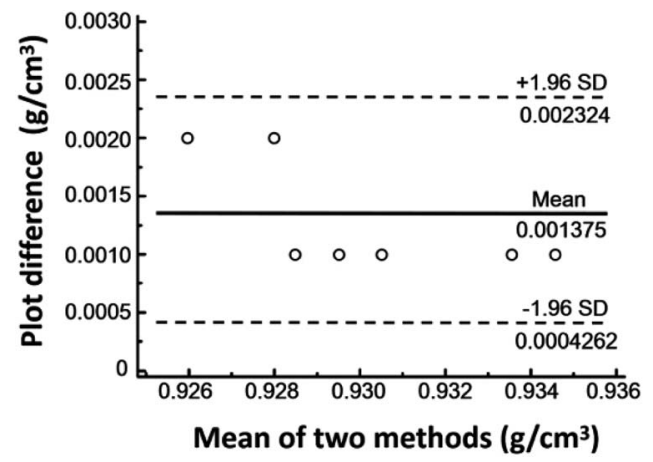

Fig. 5 Bland-Altman plot for assessing the agreement between MagLev and the traditional method (mass divided by volume). Solid black line: mean difference; dashed black line: limits of agreement (from $-1.96 \mathrm{SD}$ to $+1.96 \mathrm{SD}$ ).

two methods was evaluated using a Bland-Altman plot, which quantified the agreement between MagLev and the traditional method by constructing the limits of agreement. ${ }^{34}$ The results indicated that the method of MagLev was reliable with a high accuracy. From the above results, it can be seen that MagLev can be used for the detection of frying soybean oil with different heating time. It can be seen that MagLev can also be used to detect other frying cooking oils, showing the potential of MagLev for the quality inspection of frying cooking oils with repeated use.

(a) Certified soybean oil

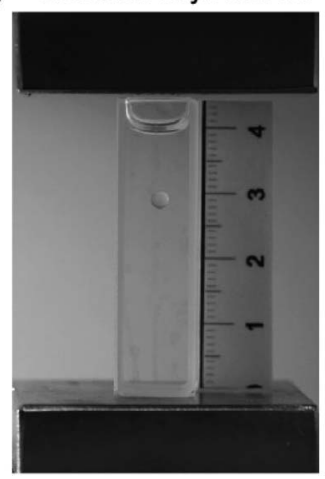

(b) Secondhand cooking oil

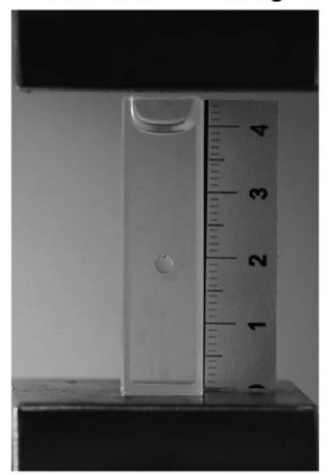

Fig. 6 Detection of secondhand cooking oil using MagLev. Photographs of the droplets of (a) certified soybean oil and (b) secondhand cooking oil levitated in the MagLev device.

\section{MagLev-based detection of secondhand cooking oil}

Secondhand cooking oil has been a major issue in China, with annual consumption of over 3 million tons. ${ }^{5}$ In this study, we demonstrated that MagLev could be potentially used for the detection of secondhand cooking oil. As shown in Fig. 6, the droplets of secondhand cooking oil and certified soybean oil were stably levitated in the solution within $15 \mathrm{~s}$ after the container was placed in the MagLev device. It can be observed that the droplet of secondhand cooking oil was levitated closer to the bottom than the droplet of certified soybean oil, indicating that the density of the secondhand cooking oil was greater than that of the certified soybean oil. The secondhand cooking oil can be differentiated from certified cooking oil based on their different levitation heights, which suggests that MagLev provides a fast, low-cost, and convenient method for distinguishing cooking oils from certified cooking oil, showing the great potential of MagLev for the quality inspection of secondhand cooking oil.

\section{Conclusions}

In this study, we demonstrated a convenient and low-cost method for the density-based detection of cooking oils using magnetic levitation (MagLev). We showed that MagLev could be used to determine the content of sesame oil in a mixed oil of sesame oil and peanut oil, with the levitation heights of the droplets of the mixed oil decreasing linearly with increasing proportion of sesame oil in the mixed oil. MagLev can also be used to determine the heating time of purchased soybean oil and pure soybean oil based on the levitation heights of the oil droplets, which decreased linearly with the heating time. We also demonstrated the potential of MagLev for differentiating secondhand cooking oil from certified cooking oils based on their different levitation heights.

For the detection of cooking oils, MagLev has several useful characteristics: (i) It is simple and inexpensive, requiring only two permanent magnets and a container filled with a paramagnetic medium. (ii) It is rapid (the detection of cooking oils can be performed within seconds). (iii) It operates without electricity. (iv) It has a relatively high accuracy. (v) It is portable and suitable for on-site detection in resource-poor settings (e.g., supermarkets and restaurants).

MagLev provides a portable and inexpensive method for the density-based detection of cooking oils such as adulterated cooking oils, repeated use of frying cooking oils, and 
secondhand cooking oil. With its high sensitivity, low cost, and convenience of operation, the use of MagLev can be extended to assess the quality of other foods, and this system may find extensive applications in quality control, food safety, and so on.

\section{Conflicts of interest}

There are no conflicts to declare.

\section{Acknowledgements}

This work was financially supported by the National Natural Science Foundation of China (21675085, 81672508, 61505077, 21505072), National Key R\&D Program of China (2017YFA0204700), China-Sweden Joint Mobility Project (51811530018), Fundamental Studies of Perovskite Solar Cells (2015CB932200), Primary Research \& Development Plan of Jiangsu Province (BE2016770), Natural Science Foundation of Jiangsu Province for Distinguished Young Scholars (BK20170042, BK20170041), Key University Science Research Project of Jiangsu Province (16KJA180004).

\section{References}

1 E. Kuraya, S. Nagatomo, K. Sakata, D. Kato, O. Niwa, T. Nishimi and M. Kunitake, Anal. Chem., 2016, 88, 1202.

2 E. K. Kabagambe, A. Baylin, A. Ascherio and H. Campos, J. Nutr., 2016, 135, 2674.

3 R. Y. Wang, Journal of Heilongjiang Grain, 2017, 05, 11.

4 R. Y. Wang, Science and Technology of Cereals, Oils and Foods, 2017, 3, 29.

5 Z. Liu, S. Yu, S. Xu, Z. Bing and W. Xu, ACS Omega, 2017, 2, 8401.

6 Y. Y. Liu, L. J. Sun, Y. S. Li, D. Zhang, Z. W. Song, C. Zhang, X. Y. Pan, J. L. Li and Z. Y. Wang, J. Food Process Eng., 2018, 7, e12887.

7 H. X. Feng, Y. Li, X. N. Sui, B. K. Qi, Z. J. Wang, L. Z. Jiang and W. M. Cao, Trans. Chin. Soc. Agric. Eng., 2016, 32, 309.

8 G. R. Takeoka, G. H. Full and L. T. Dao, J. Agric. Food Chem., 1997, 45, 3244.

9 M. Hajimahmoodi, H. Y. Vander, N. Sadeghi, B. Jannat, M. R. Oveisi and S. Shahbazian, Talanta, 2005, 66, 1108.

10 M. Paolini, L. Bontempo and F. Camin, Talanta, 2017, 174, 38.

11 J. V. B. Freitas, E. G. A. Filho, L. M. A. Silva, G. J. Zocolo, E. S. Brito and N. V. Gramosa, Talanta, 2018, 180, 676.

12 A. Saba, F. Mazzini, A. Raffaelli, A. Mattei and P. Salvadori, J. Agric. Food Chem., 2005, 53, 4867.

13 J. E. Spangenberg and N. Ogrinc, Food Chem., 2001, 49, 1534.

14 M. H. Gordon, C. Covell and N. Kirsch, J. Am. Oil Chem. Soc., 2001, 78, 621 .
15 G. Fragaki, A. Spyros, G. Siragakis, A. Emmanuel Salivaras and P. Dais, J. Agric. Food Chem., 2005, 53, 2810.

16 G. Vigli, A. Philippidis, A. Spyros and P. Dais, J. Agric. Food Chem., 2003, 51, 5715.

17 T. Mavromoustakos, M. Zervou, G. Bonas, A. Kolocouris and P. Petrakis, J. Am. Oil Chem. Soc., 2000, 77, 405.

18 S. C. Ge, S. N. Semenov, A. A. Nagarkar, J. Milette, D. C. Christodouleas, 1. Yuan and G. M. Whitesides, J. Am. Chem. Soc., 2017, 139, 18688.

19 K. A. Mirica, S. S. Shevkoplyas, S. T. Phillips, M. Gupta and G. M. Whitesides, J. Am. Chem. Soc., 2009, 131, 10049.

20 C. Matheys, N. Tumanova, T. Leyssens and A. S. Myerson, Cryst. Growth Des., 2016, 16, 5549.

21 F. Ilievski, K. A. Mirica, A. K. Ellerbee and G. M. Whitesides, Soft Matter, 2011, 7, 9113.

22 K. A. Mirica, F. Ilievski, A. K. Ellerbee, S. S. Shevkoplyas and G. M. Whitesides, Adv. Mater., 2011, 23, 4134.

23 M. R. Lockett, K. A. Mirica, C. R. Mace, R. D. Blackledge and G. M. Whitesides, J. Forensic Sci., 2013, 58, 40.

24 J. W. Hennek, A. Nemiroski, A. B. Subramaniam, D. K. Bwambok, D. Yang, D. V. Harburg, S. Tricard, A. K. Ellerbee and G. M. Whitesides, Adv. Mater., 2015, 27, 1587.

25 K. A. Mirica, S. T. Phillips, C. R. Mace and G. M. Whitesides, J. Agric. Food Chem., 2010, 58, 6565.

26 R. Amin, S. Knowlton, B. Yenilmez, A. Hart, A. Joshib and S. Tasoglu, RSC Adv., 2016, 6, 93922.

27 O. Sarigil, M. Anil-Inevi, E. Yilmaz, G. Mese, C. Tekin and E. Ozcivici, Analyst, 2019, 144, 2942.

28 T. P. Burg, M. Godin, S. M. Knudsen, W. Shen, G. Carlson, J. S. Foster, K. Babcock and S. R. Manalis, Nature, 2007, 446, 1066.

29 N. G. Durmus, H. C. Tekin, S. Guven, K. Sridhar, A. A. Yildiz, G. Calibasi, I. Ghiran, R. W. Davis, L. M. Steinmetz and U. Demirci, Proc. Natl. Acad. Sci. U. S. A., 2015, 112, E3661.

30 V. Muller, J. M. Sousa, H. C. Koydemir, M. Veli, D. Tseng, L. Cerqueira, A. Ozcan, N. F. Azevedo and F. Westerlund, RSC Adv., 2018, 8, 36493.

31 I. Banerjee, T. Salih, H. Ramachandraiah, J. Erlandsson, T. Pettersson, A. C. Araujo, M. Karlssonc and A. Russom, RSC Adv., 2017, 7, 35048.

32 P. S. Landis and R. H. Carver, J. Am. Oil Chem. Soc., 1984, 61, 1879.

33 Z. M. Zarins, R. K. Willich and R. O. Feuge, J. Am. Oil Chem. Soc., 1983, 60, 2007.

34 S. L. Yu, W. Y. Zhou, X. Q. Cheng, Q. H. Meng, H. L. Li, L. A. Hou, J. Lu, S. W. Xie, Q. Cheng, C. B. Zhang and L. Qiu, Ann. Lab. Med., 2019, 39, 381. 